\title{
Evaluation of Pathologic Complete Response in Breast Cancer Patients Treated with Neoadjuvant Chemotherapy: Experience in a Single Institution over a 10-Year Period
}

\author{
Misun Choi · Yeon Hee Park ${ }^{1}$ \\ Jin Seok Ahn ${ }^{1}$. Young-Hyuck Im $^{1}$ \\ Seok Jin $\mathrm{Nam}^{2}$. Soo Youn Cho \\ Eun Yoon Cho \\ Department of Pathology and Translational \\ Genomics, ${ }^{1}$ Division of Hematology-Oncology, \\ Department of Medicine, ${ }^{2}$ Department of \\ Surgery, Samsung Medical Center, \\ Sungkyunkwan University School of Medicine, \\ Seoul, Korea
}

Received: September 4, 2016

Revised: October 4, 2016

Accepted: October 5, 2016

Corresponding Author

Eun Yoon Cho, MD, PhD

Department of Pathology and

Translational Genomics, Samsung Medical Center, Sungkyunkwan University School of Medicine,

81 Irwon-ro, Gangnam-gu, Seoul 06351, Korea

Tel: +82-2-3410-2796

Fax: +82-2-3410-0025

E-mail: eunyoon.cho@samsung.com

Soo Youn Cho, MD, PhD

Department of Pathology and

Translational Genomics, Samsung Medical Center, Sungkyunkwan University School of Medicine,

81 Irwon-ro, Gangnam-gu, Seoul 06351, Korea

Tel: +82-2-3410-2817

Fax: +82-2-3410-0025

E-mail: sooyoun.cho@samsung.com
Background: Pathologic complete response (pCR) after neoadjuvant chemotherapy (NAC) has been associated with favorable clinical outcome in breast cancer patients. However, the possibility that the prognostic significance of $\mathrm{pCR}$ differs among various definitions has not been established. Methods: We retrospectively evaluated the pathologic response after NAC in 353 breast cancer patients and compared the prognoses after applying the following different definitions of pCR: ypTO/is, ypTO, ypTO/is ypNO, and ypTO ypNO. Results: pCR was significantly associated with improved distant disease-free survival (DDFS) regardless of the definition (ypTo/is, $p=.002$; ypT0, $p=.008$; ypT0/is ypNO, $p<.001$; ypT0 ypN0, $p=.003$ ). Presence of tumor deposits of any size in the lymph nodes (LNs; ypN $\geq 0(i+))$ was associated with worse DDFS (ypT0 ypN0 vs ypT0 $y p N \geq 0(i+), p=.036$ and ypTO/is ypNO vs ypTO/is ypN $\geq 0(i+), p=.015)$, and presence of isolated tumor cells was associated with decreased overall survival (OS; ypTO/is ypNO vs ypTO/is ypNO(i+), $\mathrm{p}=.013)$. Residual ductal carcinoma in situ regardless of $\mathrm{LN}$ status showed no significant difference in DDFS or OS (DDFS: ypTO vs ypTis, $p=.373$ and ypT0 ypN0 vs ypTis ypN0, $p=.462$; OS: ypT0 vs ypTis, $p=.441$ and ypT0 ypN0 vs ypTis ypN0, $p=.758)$. In subsequent analysis using ypT0/is ypNO, pCR was associated with improved DDFS and OS in triple-negative tumors $(p<$ .001 and $p=.003$, respectively). Conclusions: Based on our study results, the prognosis and rate of $\mathrm{pCR}$ differ according to the definition of $\mathrm{pCR}$ and $\mathrm{ypTO} / \mathrm{is}$ ypNO might be considered a more preferable definition of $\mathrm{pCR}$.

Key Words: Breast neoplasm; Pathologic complete response; Neoadjuvant chemotherapy
Neoadjuvant chemotherapy (NAC) is used as a standard therapy for inflammatory and inoperable locally advanced breast cancers. ${ }^{1}$ NAC may shrink the extent of the tumor and provide prognostic information to test treatment response. ${ }^{2}$ Pathologic complete response (pCR) after NAC is associated with improved prognosis in breast cancer and therefore is used as a surrogate of clinical outcome; ${ }^{3}$ however, the definitions of $\mathrm{pCR}$ have not been standardized, rendering interpretation of NAC data challenging. ${ }^{3-5}$

Differences among definitions of $\mathrm{pCR}$ are based on the inclu- sion of lymph node (LN) status and ductal carcinoma in situ (DCIS). The NSABP B-18 trials showed that patients with ypT0/ is had a better 5-year disease-free survival than patients with residual invasive disease in the breast, ${ }^{6,7}$ and several subsequent trials employed ypT0/is as the primary endpoint. ${ }^{8-11}$ However, several studies showed that residual tumors in LNs implied worse prognosis regardless of residual tumors in the breast. ${ }^{3,12-15}$ Isolated tumor cells (ITCs) in LNs after NAC are designated as non-pCR by the American Joint Committe on Cancer TNM; ${ }^{16}$ however, 
sufficient evidence is lacking to support this recommendation. Including residual DCIS in pCR is another controversial issue regarding the definition of $\mathrm{pCR},{ }^{3,17}$ The pooled analysis of 12 neoadjuvant randomized trials by the Collaborative Trials in Neoadjuvant Breast Cancer (CTNeoBC) showed that event-free survival and overall survival (OS) of patients with no tumor cells in the breast (ypT0 ypN0) were comparable to those of patients with residual DCIS (ypT0/is ypN0). ${ }^{12}$ Conversly, in the trials by the German Breast Group and Arbeits gemeinschaft Gynäkologische Onkologie-Breast Group (GBG and AGO-B), patients with ypTis ypN0 had a worse event-free survival than patients with ypT0 ypN0. ${ }^{3}$ However, the analysis conducted at MD Anderson Cancer Center showed no difference in survival between patients with ypT0 ypTN0 and ypTis ypTN0. ${ }^{17}$

Therefore, the previously proposed definitions of $\mathrm{pCR}$ can be divided into two main categories, assessment of pathologic response after NAC in the breast only or in both the breast and LNs. For example, the NSABP-B18 defined pCR as absence of residual invasive tumor cells in the breast (ypT0/is), and CT$\mathrm{NeoBC}$ and residual cancer burden proposed by the study conducted at MD Anderson Cancer Center defined pCR as no residual invasive tumor cells not only in the breast but also in the LNs (ypT0/is ypN0). In contrast, the Japanese Breast Cancer Society (JBCS) defined pCR as complete disappearance of tumor cells including DCIS in the breast (ypT0), and the GBG and AGO-B defined it as no residual tumor cells in the breast as well as in the LNs (ypT0 ypN0). ${ }^{3,6,7,18,19}$

Molecular intrinsic subtypes of breast cancer have important prognostic value..$^{20}$ Due to the infeasibility of this classification in routine practice, the simplified classification based on immunohistochemical (IHC) results of estrogen receptor (ER), progesterone receptor (PR), and human epidermal growth factor receptor 2 (HER2) can be used to categorize substitutes, classifying ER/PR+HER2 - as luminal A, ER/PR+HER2 + as luminal B, ER/PR-HER 2 + as HER2-positive and ER/PR-HER2- as triple-negative (TN) tumors. ${ }^{21}$ These IHC classifications also have prognostic value similar to those of molecular intrinsic subtypes. ${ }^{22}$ Thus, assessment of pCR according to subtype might provide additional prognostic information.

Different definitions of $\mathrm{pCR}$ can result in different prognosis. Defining the criteria of pCR that better predict clinical outcome would be important. Therefore, in this study, the prognostic significance of different definitions of $\mathrm{pCR}$ were compared and the prognostic significance of $\mathrm{LN}$ status, ITCs in the LN, residual DCIS and subtypes were further investigated.

\section{MATERIALS AND METHODS}

\section{Study population}

We retrospectively retrieved data from 353 individual patients from electronic medical records of Samsung Medical Center in Seoul, Korea, from January 2004 to December 2013. Patients treated with anthracycline and taxane-based NAC and who subsequently underwent surgery with curative intent for primary breast cancer were included. Patients who had histologically confirmed distant metastasis at the time of diagnosis and who were diagnosed with inflammatory carcinoma were excluded. This study was approved by the Institutional Review Board of Samsung Medical Center, and the requirement for informed consent was waived.

Baseline studies including clinical examination, mammography, ultrasound, and magnetic resonance imaging were performed to assess the extent of primary tumor in the breast and LNs. All patients were diagnosed with breast cancer based on core needle biopsies, and LN metastasis was confirmed using core needle biopsy or fine-needle aspiration. All patients were treated with four or six cycles of anthracycline and taxane-based regimen at 3-week intervals, including adriamycin with docetaxel, adriamycin with cyclophosphamide plus docetaxel (AC-T), or AC-T plus trastuzumab. Patients with hormonal receptor-positive tumors received adjuvant endocrine therapy for at least 5 years after surgery. Patients with HER2-overexpressing and/or amplified tumors received neoadjuvant trastuzumab plus chemotherapy followed by adjuvant trastuzumab. Local and regional recurrence was confirmed either histologically or cytologically.

\section{Histologic review}

Core biopsies before NAC and surgical specimens obtained after NAC were reviewed. The largest size of tumors, histologic type, histologic grade, lymphovascular invasion (LVI), proportion of DCIS, number of positive LNs, size of the largest metastasis, and treatment response in breast and LN were evaluated. Tumor size and extent in breast and LNs were assessed according to the recommendation proposed by Provenzano et al. ${ }^{23}$ Histologic type was defined in accordance with the World Health Organization classification, ${ }^{24}$ and histologic grade was classified using the modified Scarff-Bloom-Richardson grading system. ${ }^{25}$

To compare the prognostic impact of the $\mathrm{pCR}$ components defined previously, all patients were subdivided into the following subgroups according to TNM: ${ }^{16}$ (1) no residual invasive tumor cells in the breast, ypT0/is, (2) no residual invasive tumor cells in the breast or $\mathrm{LNs}$, ypT0/is ypN0, (3) no residual invasive tumor 
cells or DCIS in the breast, ypT0, and (4) no residual invasive tumor cells or DCIS in the breast and LNs, ypT0 ypN0.

ER, PR, and HER2 were assessed on both core biopsies and surgical specimens. ER and PR were considered positive only when greater than or equal to $1 \%$ of tumor cells showed nuclear staining. HER2 was positive if tumor cells showed $3+$ by IHC or $2+$ by IHC with amplification using silver in situ hybridization. ${ }^{26}$ To assess the prognostic impact of pCR on intrinsic subtypes of breast cancer, all patients were classified into four subtypes according to the IHC results as follows: ER/PR+HER2-

Table 1. Baseline clinicopathologic characteristics and pCR rates according to definition

\begin{tabular}{|c|c|c|c|c|c|c|c|c|c|}
\hline \multirow{2}{*}{ Variable } & \multirow{2}{*}{ №. (\%) } & \multicolumn{2}{|c|}{ ypTO/is } & \multicolumn{2}{|c|}{ yрT0 } & \multicolumn{2}{|c|}{ ypTO/is ypNO } & \multicolumn{2}{|c|}{ ypTO ypNO } \\
\hline & & No. (\%) & $p$-value & No. (\%) & $p$-value & No. (\%) & $p$-value & No. $(\%)$ & $p$-value \\
\hline All patients & $353(100)$ & $86(24.4)$ & & $50(14.2)$ & & $62(17.6)$ & & $40(11.3)$ & \\
\hline Age (yr) & & & .192 & & .284 & & .152 & & .308 \\
\hline Median & 44.0 & 45.5 & & 45.5 & & 46.5 & & 45.5 & \\
\hline Range & $22-68$ & $22-64$ & & $22-64$ & & $22-64$ & & $22-64$ & \\
\hline Menopause & & & .246 & & .372 & & .328 & & .432 \\
\hline Pre & $268(75.9)$ & $61(22.8)$ & & $35(13.1)$ & & $44(16.4)$ & & $28(10.4)$ & \\
\hline Post & $85(24.1)$ & $25(29.4)$ & & $15(17.6)$ & & $18(21.2)$ & & $12(14.1)$ & \\
\hline Tumor size before NAC (cm) & & & .001 & & .001 & & .036 & & .058 \\
\hline Median & 4.6 & 3.75 & & 3.45 & & 4.0 & & 3.9 & \\
\hline Range & $0.7-11.0$ & $1.1-11.0$ & & $1.1-11.0$ & & $0.7-10.0$ & & $1.1-11.0$ & \\
\hline Clinical N stage & & & .190 & & .739 & & .193 & & .533 \\
\hline $\mathrm{cN} 1$ & $10(2.8)$ & $5(50.0)$ & & $2(20.0)$ & & $4(40.0)$ & & $2(20.0)$ & \\
\hline $\mathrm{cN} 2$ & $61(17.3)$ & 16 (26.2) & & $11(18.0)$ & & $13(21.3)$ & & $9(14.8)$ & \\
\hline $\mathrm{cN} 3$ & $178(50.4)$ & 38 (21.3) & & $23(12.9)$ & & $29(16.3)$ & & $20(11.2)$ & \\
\hline $\mathrm{cN} 4$ & $104(29.5)$ & 27 (26.0) & & $14(13.5)$ & & $16(15.4)$ & & $9(8.7)$ & \\
\hline Lymph node metastasis after NAC & & & $<.001$ & & $<.001$ & & $<.001$ & & $<.001$ \\
\hline No & $135(38.2)$ & $62(45.9)$ & & 40 (29.6) & & $62(45.9)$ & & $40(29.6)$ & \\
\hline Yes & $218(61.8)$ & $24(11.0)$ & & $10(4.6)$ & & 0 & & 0 & \\
\hline Histologic type & & & .017 & & .143 & & .056 & & .233 \\
\hline Ductal & $323(91.5)$ & 85 (26.3) & & 49 (15.2) & & $61(18.9)$ & & $39(12.1)$ & \\
\hline Lobular & $9(2.5)$ & $1(11.1)$ & & $1(11.1)$ & & $1(11.1)$ & & $1(11.1)$ & \\
\hline Others & $21(5.9)$ & 0 & & 0 & & 0 & & 0 & \\
\hline Histologic grade of pre-NAC tumors & & & $<.001$ & & $<.001$ & & $<.001$ & & $<.001$ \\
\hline 1 & $27(7.6)$ & $1(3.7)$ & & 0 & & 0 & & 0 & \\
\hline 2 & $148(41.9)$ & 18 (12.2) & & $8(5.4)$ & & $12(8.1)$ & & $6(4.1)$ & \\
\hline 3 & $178(50.4)$ & $67(37.6)$ & & $42(23.6)$ & & $50(28.1)$ & & 34 (19.1) & \\
\hline Lymphovascular invasion & & & $<.001$ & & $<.001$ & & $<.001$ & & $<.001$ \\
\hline No & $223(63.2)$ & $83(37.2)$ & & $47(21.1)$ & & $62(27.8)$ & & $40(17.9)$ & \\
\hline Yes & $130(36.8)$ & $3(2.3)$ & & $3(2.3)$ & & 0 & & 0 & \\
\hline ER status & & & $<.001$ & & $<.001$ & & $<.001$ & & $<.001$ \\
\hline Negative & $167(47.3)$ & $68(40.7)$ & & $43(25.7)$ & & $48(28.7)$ & & $34(20.4)$ & \\
\hline Positive & $186(52.7)$ & $18(9.7)$ & & $7(3.8)$ & & $14(7.5)$ & & $6(3.2)$ & \\
\hline PR status & & & $<.001$ & & $<.001$ & & $<.001$ & & $<.001$ \\
\hline Negative & $214(60.6)$ & $72(33.6)$ & & $44(20.6)$ & & $53(24.8)$ & & 35 (16.4) & \\
\hline Positive & $139(39.4)$ & $14(10.1)$ & & $6(4.3)$ & & $9(6.5)$ & & $5(3.6)$ & \\
\hline HER2 status & & & .027 & & .395 & & .019 & & .714 \\
\hline Negative & $255(72.2)$ & 54 (21.2) & & $39(15.3)$ & & 37 (14.5) & & 30 (11.8) & \\
\hline Positive & $98(27.8)$ & 32 (32.7) & & 11 (11.2) & & 25 (25.5) & & 10 (10.2) & \\
\hline Subgroups & & & $<.001$ & & $<.001$ & & $<.001$ & & $<.001$ \\
\hline Luminal A-like & $120(34.0)$ & $6(5.0)$ & & $3(2.5)$ & & $5(4.2)$ & & $2(1.7)$ & \\
\hline Luminal B-like & $71(20.1)$ & 14 (19.7) & & $5(7.0)$ & & $10(14.1)$ & & $5(7.0)$ & \\
\hline HER2-positive & 55 (15.6) & $23(41.8)$ & & $8(14.5)$ & & 18 (32.7) & & $7(12.7)$ & \\
\hline Triple-negative & $107(30.3)$ & $43(40.2)$ & & 34 (31.8) & & $29(27.1)$ & & 26 (24.3) & \\
\hline
\end{tabular}

pCR, pathologic complete response; NAC, neoadjuvant chemotherapy; ER, estrogen receptor; PR, progesterone receptor; HER2, human epidermal growth factor receptor 2; Luminal A-like, ER/PR+HER2- tumors with histologic grade 1 or 2; Luminal B-like, ER/PR+HER2- tumors with histologic grade 3 or ER/ PR+HER2+ tumors; HER2-positive, ER/PR-HER2+ tumors; Triple-negative, ER/PR/HER2- tumors. 
with histologic grade 1 or 2 tumors as luminal A-like; ER/ PR +HER2- with histologic grade 3 tumors or ER/PR/HER2+ tumors as luminal B-like; ER/PR-HER2 + tumors as HER2positive; and ER/PR/HER2- tumors as TN. ${ }^{3,27}$

\section{Statistical analysis}

Distant disease-free survival (DDFS) and OS were defined as the time between the date of initial diagnosis to the date of distant recurrence or the date of death from any cause, respectively. The significance of differences in baseline variables was analyzed using two-sided $\chi^{2}$, Fisher exact or Mann-Whitney U test as appropriate. DDFS and OS were plotted using the Kaplan-Meier productlimit method, and the log-lank p-value was calculated. To assess the prognostic impact of the pCR component, hazard ratios
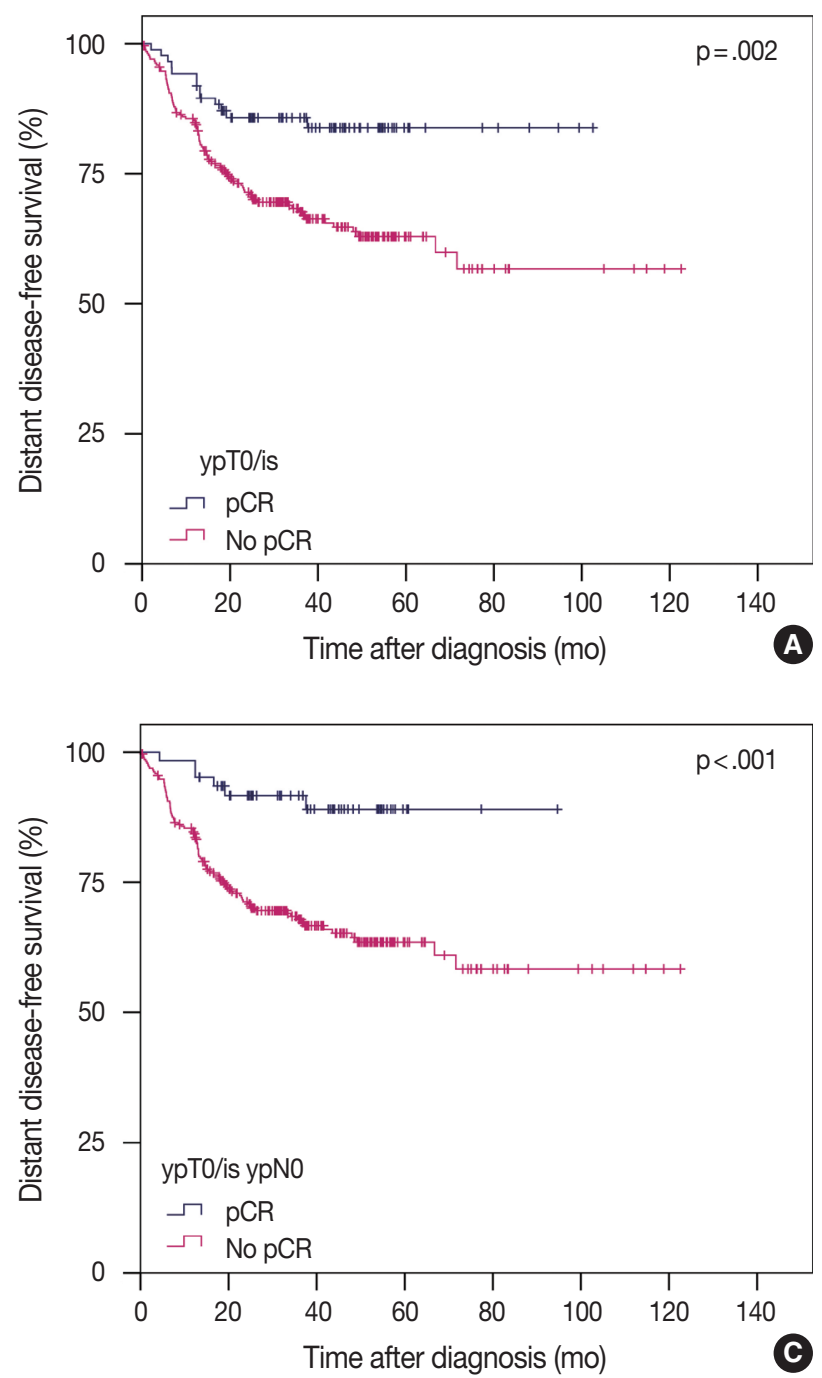

(HRs), 95\% confidence intervals and p-value were calculated using Cox proportional hazards model. All tests were two sided, and a p-value less than .05 was considered statistically significant. Statistical analyses were performed using the SPSS statistical software ver. 20.0 (IBM Corp., Armonk, NY, USA).

\section{RESULTS}

\section{Patient characteristics}

Median age at diagnosis was 44.0 years (range, 22 to 68 years). Median size of tumor was $4.6 \mathrm{~cm}$ (range, 0.7 to $11.0 \mathrm{~cm}$ ) before NAC and $1.5 \mathrm{~cm}$ (range, 0.0 to $13.0 \mathrm{~cm}$ ) after NAC. Most patients $(282 / 353,79.9 \%)$ had cN2 or N3 nodal status before NAC, and median number of positive LNs after NAC
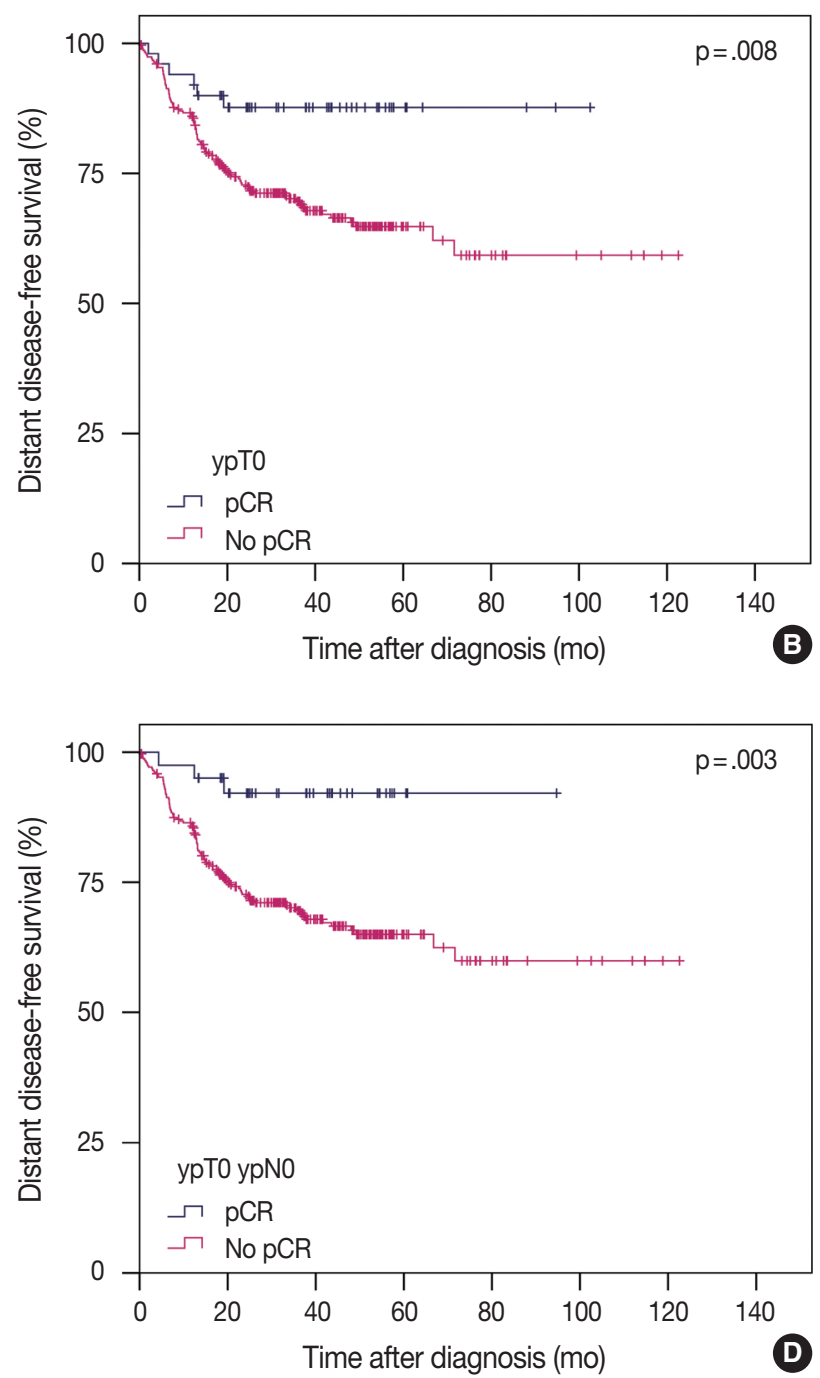

Fig. 1. Survival analysis according to definition of pCR. DDFS and OS according to ypTO/is definition of pCR (A, B), ypTO (C, D), ypTO/is ypNO (E, F), and ypTO ypNO (G, H). pCR, pathologic complete response; DDFS, distant disease-free survival; OS, overall survival. (Continued to the next page) 

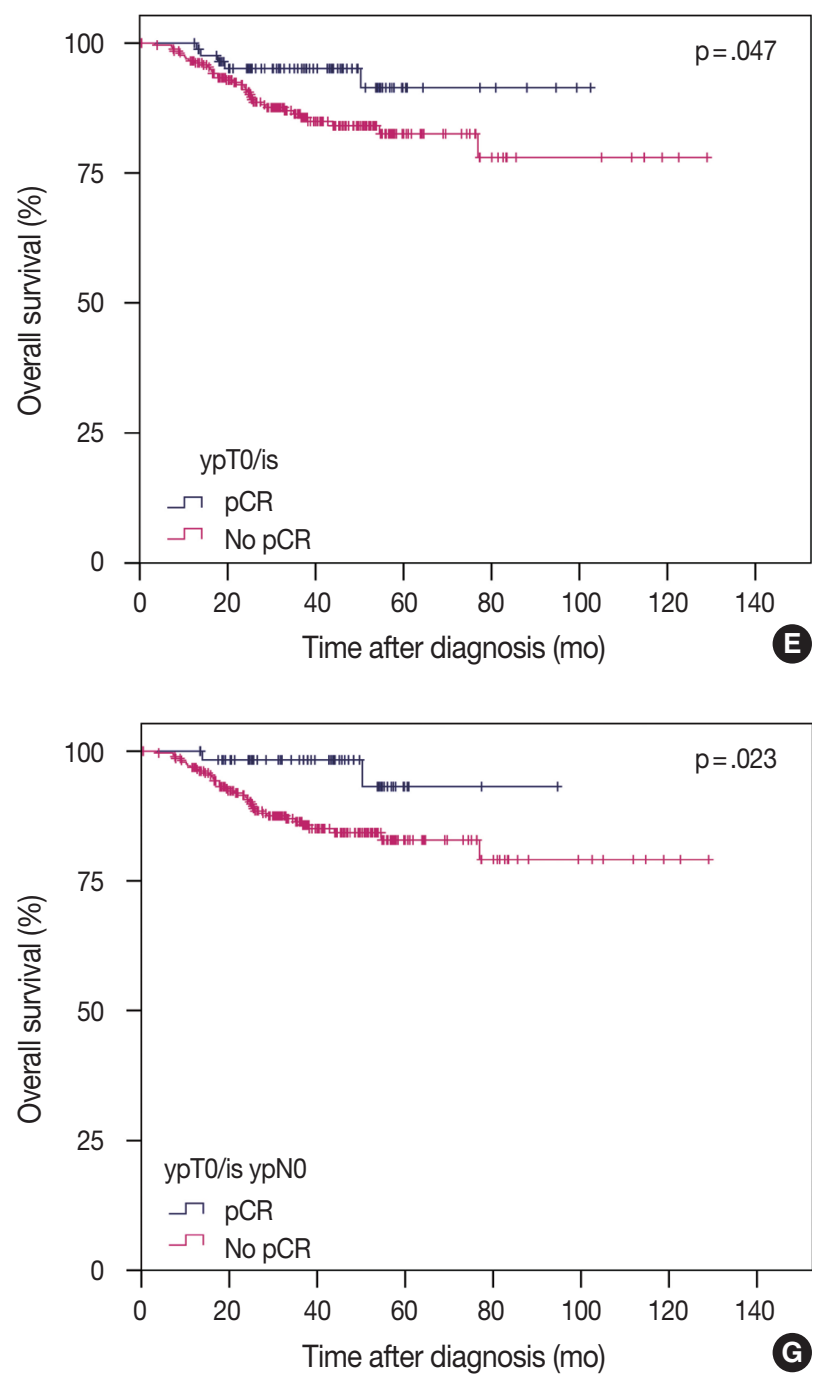

Fig. 1. (Continued from the previous page)

was 1 (range, 0 to 39). Baseline clinicopathologic characteristics and corresponding $\mathrm{pCR}$ rates according to definition of $\mathrm{pCR}$ are summarized in Table 1. In brief, histologic grade, LVI, presence of metastatic $\mathrm{LN}$, and hormonal receptor status showed significant correlation with $\mathrm{pCR}$ rate according to definition. Patients with high histologic grade tumors, no LVI, no metastatic LN, and negative hormonal receptor status tended to have higher $\mathrm{PCR}$ rates. HER2-positive and TN tumors showed significantly higher pCR rates than luminal A-like and luminal B-like tumors regardless of the pCR definition. The median follow-up time of 353 patients was 36.5 months (range, 0.4 to 129.0 months). During this period, 101 patients (28.6\%) had a relapse and $41(11.6 \%)$ died. The 5 -year DDFS was $68.0 \%$, and OS was $84.8 \%$.

\section{Correlation between $\mathrm{pCR}$ and survival according to definition}

According to the four definitions of pCR, 86 (24.4\%) patients
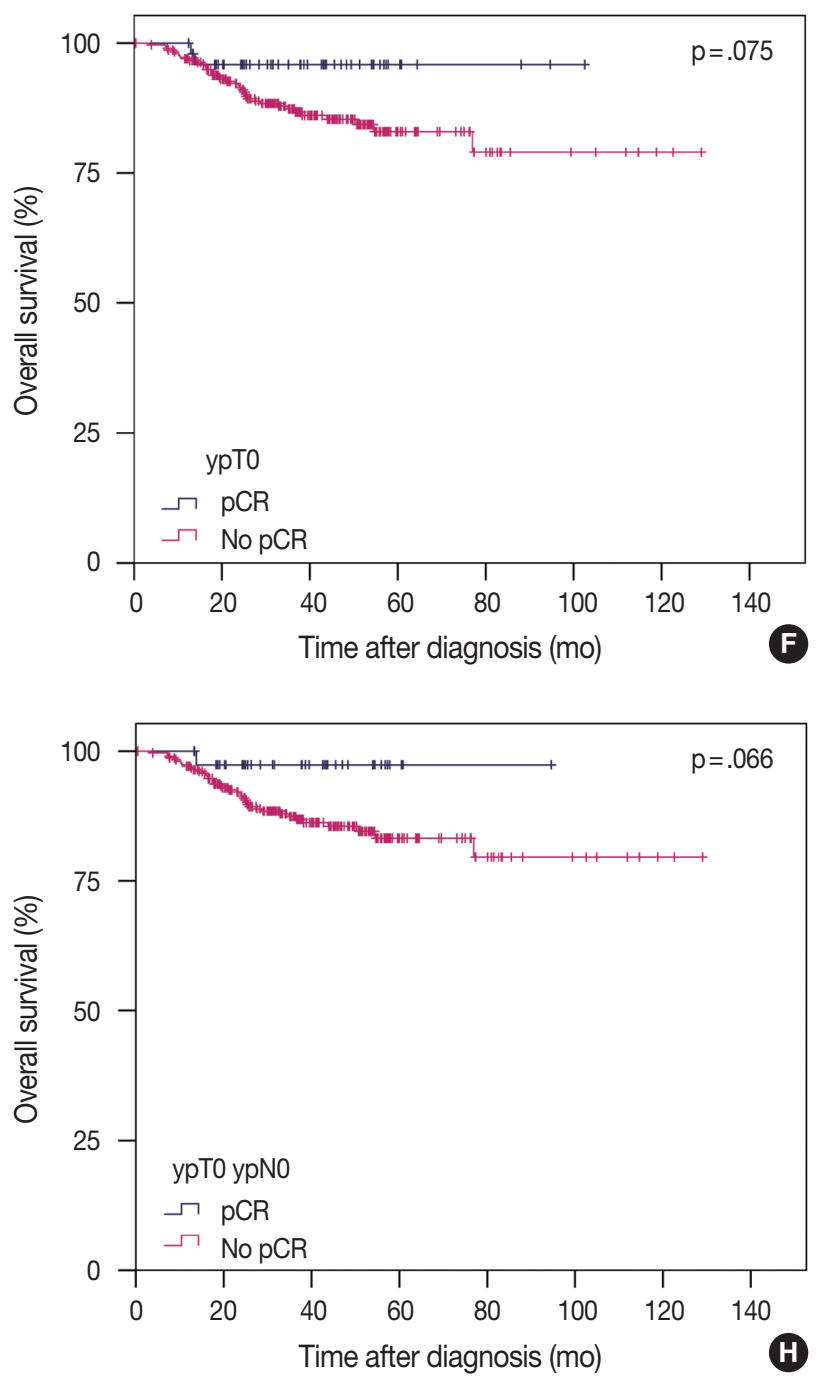

were diagnosed as ypT0/is, $50(14.2 \%)$ as ypT0, $62(17.6 \%)$ as ypT0/is ypN0, and $40(11.3 \%)$ as ypT0 ypN0. Patients who achieved pCR showed significantly better DDFS than patients who did not (Fig. 1A, C, E, G). Similarly, patients who achieved pCR also tended to have better OS than patients who did not, but this difference was not statistically significant when ypT0 and ypT0 ypN0 were used as the pCR definitions (Fig. 1B, D, F, H). HRs for DDFS and OS increased sequentially as follows: ypT0/is, ypT0, ypT0/is ypN0, and ypT0 ypN0 (Table 2).

\section{Prognostic significance of $L N$ status}

In the ypT0 subgroup ( $\mathrm{n}=50), 10$ patients $(20.0 \%)$ had tumor deposits in the LNs $(\mathrm{ypN} \geq 0(\mathrm{i}+))$ and experienced worse DDFS than patients with no metastatic LNs (5-year DDFS: ypT0 ypN0, 92.1\%; ypT0 ypN $\geq 0(i+), 68.6 \%$; $\mathrm{p}=.036)$. In the ypT0/ is subgroup ( $\mathrm{n}=86), 24$ patients (27.9\%) had residual tumor 
deposits in the LNs $(y \mathrm{pN} \geq 0(\mathrm{i}+))$ and experienced worse DDFS than patients with no metastatic LNs (5-year DDFS: ypT0/is ypN0, 89.0\%; ypT0/is ypN $\geq 0(i+), 70.2 \%$; $\mathrm{p}=.015)$. The 5-year OS for patients with ypT0 ypN $\geq 0(\mathrm{i}+)$ and $\mathrm{ypT} 0 /$ is ypN $\geq 0(\mathrm{i}+)(88.9 \%$ and $86.5 \%$, respectively) appeared worse than for patients with ypT0 ypN0 and ypT0/is ypN0 $(97.4 \%$ and

Table 2. Prognostic significance of pCR on survival according to definition

\begin{tabular}{|c|c|c|c|c|c|c|c|}
\hline \multirow{2}{*}{ Definitions of $\mathrm{pCR}$} & \multirow{2}{*}{ Total, n (\%) } & \multirow{2}{*}{$\begin{array}{c}\text { Distant metastasis, } \\
\mathrm{n}(\%)\end{array}$} & \multicolumn{2}{|l|}{ DDFS } & \multirow{2}{*}{$\begin{array}{c}\text { Died of disease, } \\
n(\%)\end{array}$} & \multicolumn{2}{|l|}{ OS } \\
\hline & & & $\mathrm{HR}^{\mathrm{a}}(95 \% \mathrm{Cl})$ & $p$-value ${ }^{a}$ & & $\mathrm{HR}^{\mathrm{a}}(95 \% \mathrm{Cl})$ & $p$-value ${ }^{a}$ \\
\hline \multicolumn{8}{|l|}{ Breast only } \\
\hline ypTO/is & $86(24.4)$ & $13(15.1)$ & $2.472(1.380-4.426)$ & .002 & $5(5.9)$ & $2.497(0.980-6.364)$ & .055 \\
\hline урт0 & $50(14.2)$ & $6(12.0)$ & 2.900 (1.270-6.618) & .011 & $2(4.0)$ & $3.373(0.814-13.969)$ & .094 \\
\hline \multicolumn{8}{|c|}{ Breast and lymph nodes } \\
\hline ypTO/is ypNO & $62(17.6)$ & $6(9.7)$ & 3.954 (1.732-9.026) & .001 & $2(3.2)$ & 4.498 (1.086-18.638) & .038 \\
\hline урTO ypNO & $40(11.3)$ & $3(7.5)$ & $4.741(1.502-14.958)$ & .008 & $1(2.5)$ & $5.277(0.725-38.398)$ & .100 \\
\hline
\end{tabular}

pCR, pathologic complete response; DDFS, distant disease-free survival; OS, overall survival; $\mathrm{HR}$, hazard ratio; $\mathrm{Cl}$, confidende interval.

aHRs and p-values are calculated from the comparison of survival in patients with or without pCR.
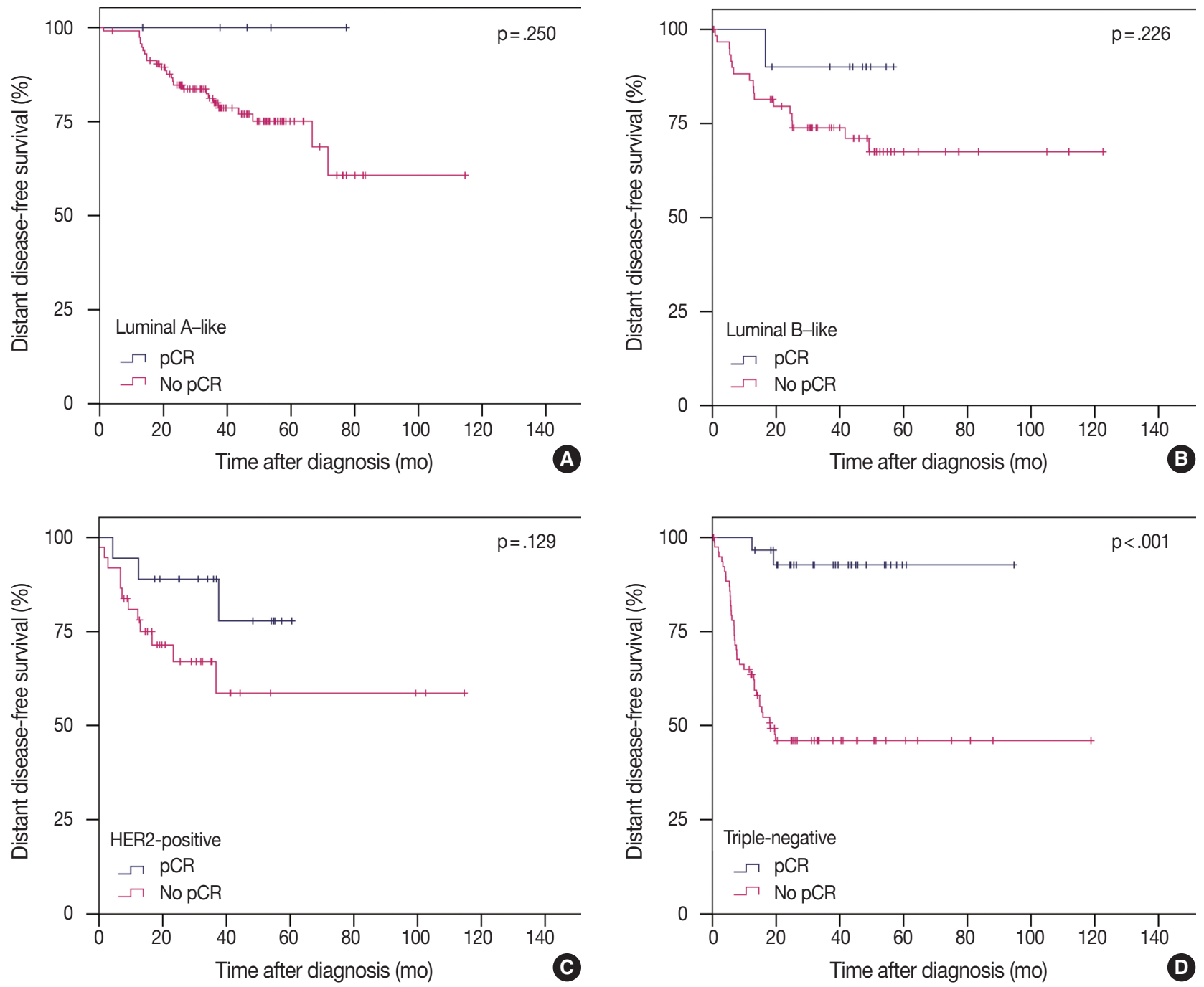

Fig. 2. (A-H) Prognosis between patients with or without $\mathrm{pCR}$ according to intrinsic subtype. $\mathrm{pCR}$, pathologic complete response; Luminal A-like, ER/PR+HER2- tumors with histologic grade 1 or 2; Luminal B-like, ER/PR+HER2- tumors with histologic grade 3 or ER/PR+HER2+ tumors; HER2-positive, ER/PR-HER2+ tumors; Triple-negative, ER/PR/HER2- tumors; ER, estrogen receptor; PR, progesterone receptor; HER2, human epidermal growth factor receptor 2. (Continued to the next page) 

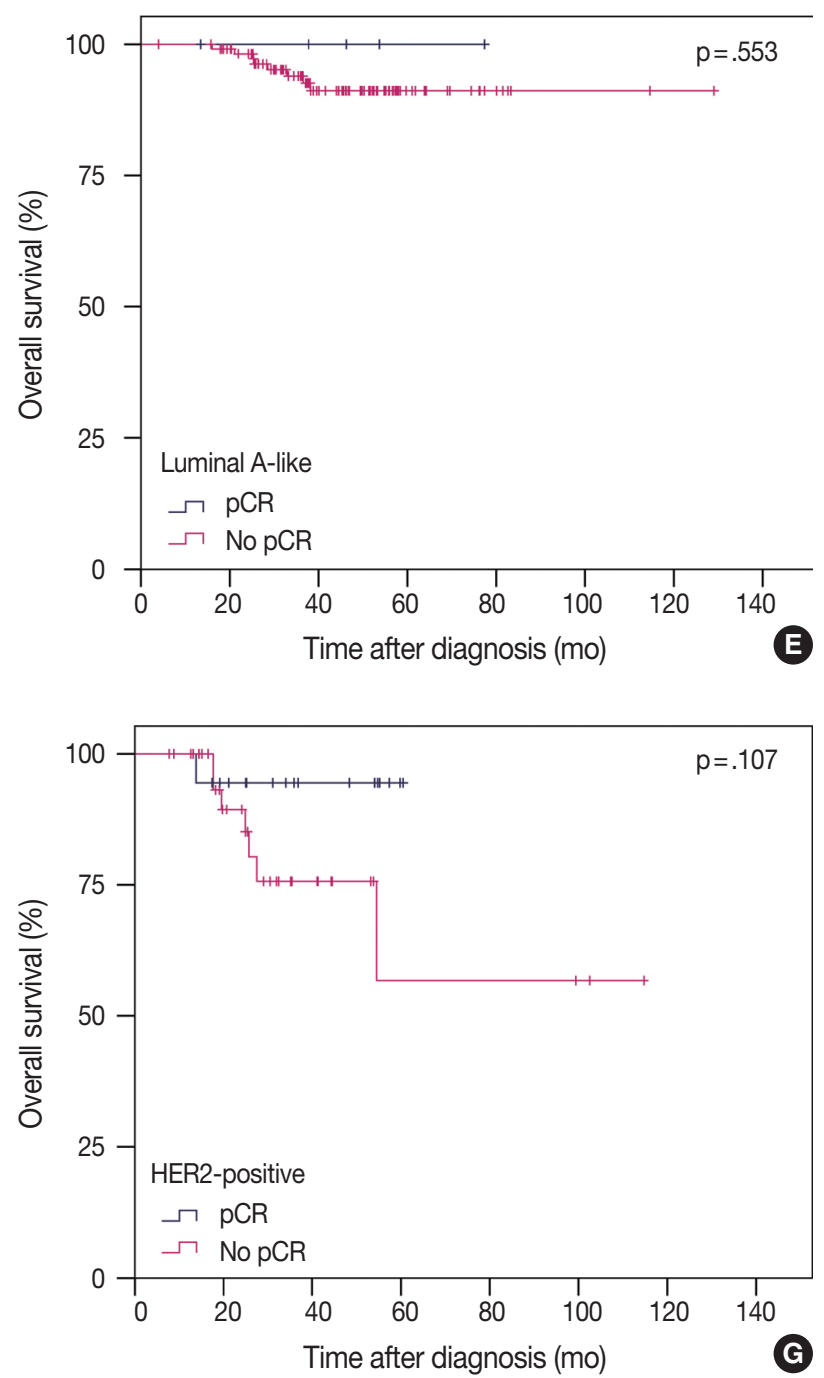

Fig. 2. (Continued from the previous page)

$93.2 \%$, respectively), but the difference was not statistically significant ( $\mathrm{p}=.236$ and $\mathrm{p}=.095$, respectively).

\section{Prognostic significance of ITCs in the LNs}

Among patients with ypT0 ypN $\geq 0(i+)(n=10), 3$ had ITCs in the LNs, and none relapsed or died. Among patients with ypT0/is ypN $\geq 0(i+)(n=24)$, five had ITCs in the LNs, and showed worse OS compared to patients with ypT0/is ypN0 (5year OS: ypT0/is ypNO(i+), 75.0\% vs ypT0/is ypN0, 93.2\%; p $=.013)$. For DDFS, patients with ITCs in LNs tended to experience worse DDFS than patients with no tumor cells in the LNs (5-year DDFS: ypT0/is ypNO(i+), $80.0 \%$ vs ypT0/is ypN0, $89.0 \% ; \mathrm{p}=.336)$.

\section{Prognostic significance of residual DCIS}

Patients with residual DCIS in the breast tended to experience
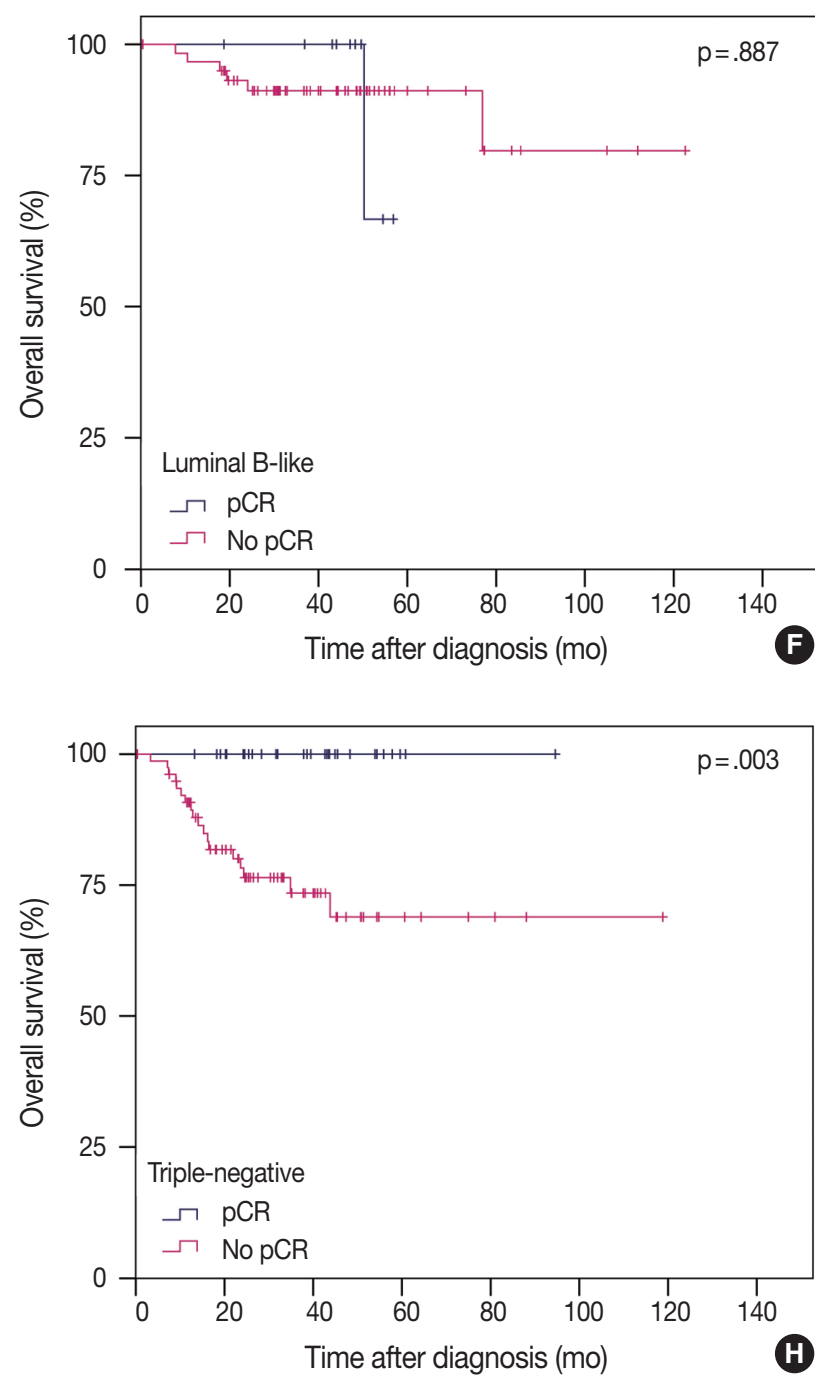

worse DDFS and OS compared with patients with no residual tumor cells in the breast (5-year DDFS: ypT0, 87.6\% and ypTis, 78.3\%; 5-year OS: ypT0, 95.8\% and ypTis, 85.5\%), but the difference was not statistically significant $(\mathrm{p}=.373$ and $\mathrm{p}=.441$, respectively). Considering LN status, patients with ypT0 ypN0 had 5-year DDFS of $92.1 \%$ and OS of $97.4 \%$, which appeared better than those of patients with ypTis ypN0 (5-year DDFS, $83.3 \%$ and 5 -year OS, $87.5 \%$ ). However, these were not statistically significant $(\mathrm{p}=.462$ and $\mathrm{p}=.758$, respectively).

Among patients who achieved $\mathrm{pCR}$ when $\mathrm{ypT} 0 /$ is was used as the definition of $\mathrm{pCR}$, the proportion of patients with residual DCIS was significantly different among IHC subtypes ( $\mathrm{p}=.001$ ); highest with HER2-positive tumors (15/23, 65.2\%) followed by luminal B-like $(9 / 14,64.3 \%)$, luminal A-like (3/6, 50\%), and lowest with TN tumors $(9 / 43,20.9 \%)$. 


\section{Prognostic significance of $\mathrm{pCR}$ in $\mathrm{IHC}$ subtypes}

To analyze the prognosis between patients with or without $\mathrm{pCR}$ according to IHC subtype, we defined $\mathrm{pCR}$ as ypT0/is ypN0 according to our study results. In TN tumors, pCR was significantly associated with improved survival in terms of both DDFS and OS. However, in luminal A-like, luminal B-like, and HER2-positive tumors, pCR showed no prognostic impact on survival (Fig. 2). In patients without pCR, HER2-positive and TN tumors showed poorer prognosis than in luminal Alike and luminal B-like tumors (Fig. 2).

\section{DISCUSSION}

To the best of our knowledge, this study is the first analysis of the prognostic significance of different pCR definitions on long-term outcome in breast cancer patients treated homogeneously with anthracycline and taxane-based NAC regimens as well as neoadjuvant trastzumab plus chemotherapy at a single institute in Korea. We compared the following four definitions of pCR; ypT0/is, ypT0, ypT0/is ypN0, and ypT0 ypN0, and the corresponding $\mathrm{pCR}$ rates were $24.4 \%, 14.2 \%, 17.6 \%$, and $11.3 \%$, respectively (Table 1 ). These rates were similar to previous studies. In a study by JBCS ( $\mathrm{n}=353)$, pCR rates of ypT0/is, ypT0, урT0/is ypN0, and yрT0 ypN0 were $20.4 \%, 9.9 \%, 18.4 \%$, and $8.2 \%$, respectively. ${ }^{28}$ In a meta-analysis by CTNeoBC ( $\mathrm{n}=$ 13,125), pCR rates of ypT0/is, ypT0/is ypN0, and ypT0 ypN0 were $22 \%, 18 \%$, and $13 \%$, respectively. ${ }^{12}$ In the study by GBG and AGO-B $(\mathrm{n}=6,377), \mathrm{pCR}$ rates of ypT0/is, ypT0/is ypN0, and ypT0 ypN0 were $22.8 \%, 19.8 \%$, and $15.0 \%$, respectively. ${ }^{3}$

In the present study, patients with $\mathrm{pCR}$, regardless of definition, had significantly better DDFS than patients without pCR. Regarding OS, however, patients with $\mathrm{pCR}$ when $\mathrm{ypT} 0 /$ is and ypT0/is ypN0 were used as pCR definitions showed significantly better survival than patients without pCR (Fig. 1). Regarding LN status, even if tumor cells were not present in the breast including DCIS, patients with residual tumor cells of any size in the LNs experienced worse DDFS than patients with no metastatic LNs. Presence of ITCs in the LNs after NAC is regarded as non-pCR by the American Joint Committee on Cancer for TNM staging. ${ }^{16}$ however, data supporting this recommendation is insufficient. Our study showed that patients with ITCs in the LNs might have poorer OS than patients with no tumor cells in the LNs. However, further studies with larger populations are warranted.

Theoretically, ypT0 ypN0 represents the strictest definition of pCR, meaning complete eradication of all tumor cells in both the breast and LNs. Thus, we compared the prognosis between ypT0 and ypTis as well as between ypT0 ypN0 and ypT0/is ypN0 and found that presence of DCIS did not result in any difference. Thus, based on the results from this study, we considered ypT0/is ypN0 the more preferable definition of $\mathrm{pCR}$. These results were consistent with previous studies by JBCS, CTNeoBC, and MD Anderson. ${ }^{12,17,28}$ But not with those of GBG and AGO-B, which suggested ypT0 ypN0 as the best definition of $\mathrm{pCR}{ }^{3}$ This discrepancy might be caused by the smaller number of patients and events in ypTis $(\mathrm{n}=36)$ and $\mathrm{ypTis}$ $\mathrm{ypN} 0(\mathrm{n}=22)$, resulting in a much lower statistical significance to show prognostic differences in this study.

Among the IHC subtypes, HER2-positive and TN tumors achieved high $\mathrm{pCR}$ rates. $\mathrm{pCR}$ was significantly correlated with DDFS and OS only in TN tumors (Fig. 2). However, in patients without pCR, HER2-positive and TN tumors showed poorer prognosis than in luminal A-like and luminal B-like tumors. These results are in agreement with the previously reported studies by Liedtke et al..$^{29}$ and Houssami et al..$^{30}$

The potential limitations of this study are as follows. First, due to the small number of patients and events, comparison between patients with $\mathrm{pCR}$ and without $\mathrm{pCR}$ using Cox proportional hazards model was not feasible. Second, because Ki-67 was not available for all patients, subtypes based on only ER, PR, and HER2 status and histologic grade might not be the same as molecular intrinsic subtypes.

In conclusion, the prognosis and rate of $\mathrm{pCR}$ varied according to definition of pCR. In our study, pCR defined as ypT0/is ypN0 was considered the most preferable. pCR could be used as a surrogate of favorable clinical outcome in TN tumors but not in luminal A-like, luminal B-like, or HER2-positive tumors.

\section{Conflicts of Interest}

No potential conflict of interest relevant to this article was reported.

\section{REFERENCES}

1. Kaufmann M, von Minckwitz G, Bear HD, et al. Recommendations from an international expert panel on the use of neoadjuvant (primary) systemic treatment of operable breast cancer: new perspectives 2006. Ann Oncol 2007; 18: 1927-34.

2. Kaufmann M, von Minckwitz G, Mamounas EP, et al. Recommendations from an international consensus conference on the current status and future of neoadjuvant systemic therapy in primary breast 
cancer. Ann Surg Oncol 2012; 19: 1508-16

3. von Minckwitz G, Untch M, Blohmer JU, et al. Definition and impact of pathologic complete response on prognosis after neoadjuvant chemotherapy in various intrinsic breast cancer subtypes. J Clin Oncol 2012; 30: 1796-804.

4. Cortazar P, Geyer CE Jr. Pathological complete response in neoadjuvant treatment of breast cancer. Ann Surg Oncol 2015; 22: 1441-6.

5. Kuroi K, Toi M, Tsuda H, Kurosumi M, Akiyama F. Issues in the assessment of the pathologic effect of primary systemic therapy for breast cancer. Breast Cancer 2006; 13: 38-48.

6. Wolmark N, Wang J, Mamounas E, Bryant J, Fisher B. Preoperative chemotherapy in patients with operable breast cancer: nine-year results from National Surgical Adjuvant Breast and Bowel Project B-18. J Natl Cancer Inst Monogr 2001; (30): 96-102.

7. Fisher B, Bryant J, Wolmark N, et al. Effect of preoperative chemotherapy on the outcome of women with operable breast cancer. J Clin Oncol 1998; 16: 2672-85.

8. Robidoux A, Tang G, Rastogi P, et al. Lapatinib as a component of neoadjuvant therapy for HER2-positive operable breast cancer (NSABP protocol B-41): an open-label, randomised phase 3 trial. Lancet Oncol 2013; 14: 1183-92.

9. Bear HD, Tang G, Rastogi P, et al. Bevacizumab added to neoadjuvant chemotherapy for breast cancer. N Engl J Med 2012; 366: 310-20.

10. Gianni L, Pienkowski T, Im YH, et al. Efficacy and safety of neoadjuvant pertuzumab and trastuzumab in women with locally advanced, inflammatory, or early HER2-positive breast cancer (NeoSphere): a randomised multicentre, open-label, phase 2 trial. Lancet Oncol 2012; 13: 25-32.

11. Baselga J, Bradbury I, Eidtmann $\mathrm{H}$, et al. Lapatinib with trastuzumab for HER2-positive early breast cancer (NeoALTTO): a randomised, open-label, multicentre, phase 3 trial. Lancet 2012; 379: 633-40.

12. Cortazar P, Zhang L, Untch M, et al. Pathological complete response and long-term clinical benefit in breast cancer: the CTNeoBC pooled analysis. Lancet 2014; 384: 164-72.

13. Corben AD, Abi-Raad R, Popa I, et al. Pathologic response and longterm follow-up in breast cancer patients treated with neoadjuvant chemotherapy: a comparison between classifications and their practical application. Arch Pathol Lab Med 2013; 137: 1074-82.

14. Rouzier R, Extra JM, Klijanienko J, et al. Incidence and prognostic significance of complete axillary downstaging after primary chemotherapy in breast cancer patients with $\mathrm{T} 1$ to $\mathrm{T} 3$ tumors and cytologically proven axillary metastatic lymph nodes. J Clin Oncol 2002; 20: 1304-10.

15. McCready DR, Hortobagyi GN, Kau SW, Smith TL, Buzdar AU, Balch CM. The prognostic significance of lymph node metastases after preoperative chemotherapy for locally advanced breast can- cer. Arch Surg 1989; 124: 21-5.

16. Edge SB, Byrd DR, Compton CC, Fritz AG, Greene FL, Trotti A. American Joint Committee on Cancer (AJCC) cancer staging manual. 7th ed. New York: Springer, 2009.

17. Mazouni C, Peintinger F, Wan-Kau S, et al. Residual ductal carcinoma in situ in patients with complete eradication of invasive breast cancer after neoadjuvant chemotherapy does not adversely affect patient outcome. J Clin Oncol 2007; 25: 2650-5.

18. Symmans WF, Peintinger F, Hatzis C, et al. Measurement of residual breast cancer burden to predict survival after neoadjuvant chemotherapy. J Clin Oncol 2007; 25: 4414-22.

19. Kurosumi M, Akiyama F, Iwase T, et al. Histopathological criteria for assessment of therapeutic response in breast cancer. Breast Cancer 2001; 8: 1-2.

20. Perou CM, Sorlie T, Eisen MB, et al. Molecular portraits of human breast tumours. Nature 2000; 406: 747-52.

21. Carey LA, Perou CM, Livasy CA, et al. Race, breast cancer subtypes, and survival in the Carolina Breast Cancer Study. JAMA 2006; 295: 2492-502.

22. Onitilo AA, Engel JM, Greenlee RT, Mukesh BN. Breast cancer subtypes based on ER/PR and Her2 expression: comparison of clinicopathologic features and survival. Clin Med Res 2009; 7: 4-13.

23. Provenzano E, Bossuyt V, Viale G, et al. Standardization of pathologic evaluation and reporting of postneoadjuvant specimens in clinical trials of breast cancer: recommendations from an international working group. Mod Pathol 2015; 28: 1185-201.

24. Lakhani SR, Ellis IO, Schinitt SJ, Tan PH, van de Vijver MJ. WHO classification of tumours of the breast. 4th ed. Lyon: IARC Press, 2012

25. Elston CW, Ellis IO. Pathological prognostic factors in breast cancer. I. The value of histological grade in breast cancer: experience from a large study with long-term follow-up. Histopathology 1991; 19: 40310.

26. Wolff AC, Hammond ME, Hicks DG, et al. Recommendations for human epidermal growth factor receptor 2 testing in breast cancer: American Society of Clinical Oncology/College of American Pathologists clinical practice guideline update. J Clin Oncol 2013; 31: 39974013.

27. Goldhirsch A, Wood WC, Coates AS, et al. Strategies for subtypes: dealing with the diversity of breast cancer: highlights of the St. Gallen International Expert Consensus on the Primary Therapy of Early Breast Cancer 2011. Ann Oncol 2011; 22: 1736-47.

28. Kuroi K, Toi M, Ohno S, et al. Comparison of different definitions of pathologic complete response in operable breast cancer: a pooled analysis of three prospective neoadjuvant studies of JBCRG. Breast Cancer 2015; 22: 586-95.

29. Liedtke C, Mazouni C, Hess KR, et al. Response to neoadjuvant ther- 
apy and long-term survival in patients with triple-negative breast cancer. J Clin Oncol 2008; 26: 1275-81.

30. Houssami N, Macaskill P, von Minckwitz G, Marinovich ML, Ma- mounas E. Meta-analysis of the association of breast cancer subtype and pathologic complete response to neoadjuvant chemotherapy. Eur J Cancer 2012; 48: 3342-54. 